\title{
1 Interface Verification for Multiagent Probabilistic Inference
}

\author{
Y. Xiang and X. Chen \\ University of Guelph, Guelph, Ontario, Canada
}

\begin{abstract}
Multiply sectioned Bayesian networks support representation of probabilistic knowledge in multiagent systems. To ensure exact, distributed reasoning, agent interfaces must satisfy the d-sepset condition. Otherwise, the system will behave incorrectly. We present a method that allows agents to verify cooperatively the d-sepset condition through message passing. Each message reveals only partial information on the adjacency of a shared node in an agent's local network. Hence, the method respects agent's privacy, protects agent vendors' know-how, and promotes integration of multiagent systems from independently developed agents.
\end{abstract}

\section{$1.1 \quad$ Introduction}

As the cost of computers and networking continues to drop and distributed systems are widely deployed, users are expecting more intelligent behaviors from such systems - multiagent systems (MAS) [14]. Agents in an MAS perform a set of tasks depending on the particular application domain. A common task is for a set of cooperative agents to determine what is the current state of the domain so that they can act accordingly. Agents monitoring a piece of equipment need to determine whether the equipment is functioning normally and, if not, which components have failed. Agents populating a smart house should recognize the current need of inhabitants and adjust the appliances accordingly. Similar situations arise in other domains such as cooperative design, battle field assessment, and surveillance. Often agents have only uncertain knowledge about the domain and must perform the task based on partial observations. Such a task has been termed distributed interpretation [7] by some authors. We shall refer to it as multiagent situation assessment.

Different approaches have been proposed to tackle multiagent situation assessment. Blackboard [10] offers a framework for multiagent inference and cooperation. It does not dictate how uncertain knowledge should be represented nor offers any guarantee of inference coherence. DATMS [8] and DTMS [4] offer inference frameworks based on default reasoning. Relation between BDI model and decision-tree is studied in [12]. Reasoning about the mental state of an agent from the received communication is considered by [2]. Monitoring whether a multiagent system is functioning normally by focusing on agent-relation is investigated in [5]. Emotions of agents are studied using decision theory in [3]. Proving hypotheses by agents with distributed 
knowledge using dialectical argumentation is proposed in [9]. Multiply sectioned Bayesian networks (MSBNs) [15] provide a framework where agents' knowledge can be encoded with graphical models and agent's belief can be updated by distributed, exact probabilistic reasoning. Multiagent MSBNs (MAMSBNs) are the focus of this work.

Distributed and exact inference requires that an MAMSBN observes a set of constraints [15]. When building an MAMSBN, these constraints on the knowledge representation need to be verified before inference for situation assessment takes place. Otherwise, garbage-in-garbage-out may occur and the resultant MAS will not reason correctly. When agents are autonomous and may be constructed by independent vendors (hence privacy of agents becomes an issue), verification of these constraints raises a challenge. In this work, we study verification of agent interface. We present a method that verifies the correctness of agent interfaces in an MAMSBN without compromising agent autonomy and privacy.

Section 1.2 briefly overviews MAMSBNs and introduces formal background necessary for the remainder of the paper.

\subsection{Overview of MAMSBNs}

A BN [11] $S$ is a triplet $(N, G, P)$, where $N$ is a set of domain variables, $G$ is a DAG whose nodes are labeled by elements of $N$, and $P$ is a joint probability distribution (jpd) over $N$. In an MAMSBN, a set of $n>1$ agents $A_{0}, \ldots, A_{n-1}$ populates a total universe $V$ of variables. Each $A_{i}$ has knowledge over a subdomain $V_{i} \subset V$ encoded as a Bayesian subnet $\left(V_{i}, G_{i}, P_{i}\right)$. The collection of local DAGs $\left\{G_{i}\right\}$ encodes agents' knowledge of domain dependency. Distributed and exact reasoning requires these local DAGs to satisfy some constraints [15] described below:

Let $G_{i}=\left(V_{i}, E_{i}\right)(i=0,1)$ be two graphs. The graph $G=\left(V_{0} \cup V_{1}, E_{0} \cup\right.$ $E_{1}$ ) is referred to as the union of $G_{0}$ and $G_{1}$, denoted by $G=G_{0} \sqcup G_{1}$. If each $G_{i}$ is the subgraph of $G$ spanned by $V_{i}$, we say that $G$ is sectioned into $G_{i}(i=0,1)$. Local DAGs of an MAMSBN should overlap and be organized into a hypertree.

Definition 1 Let $G=(V, E)$ be a connected graph sectioned into subgraphs $\left\{G_{i}=\left(V_{i}, E_{i}\right)\right\}$. Let the $G_{i}$ s be organized as a connected tree $\Psi$, where each node is labeled by $a G_{i}$ and each link between $G_{k}$ and $G_{m}$ is labeled by the interface $V_{k} \cap V_{m}$ such that for each $i$ and $j, V_{i} \cap V_{j}$ is contained in each subgraph on the path between $G_{i}$ and $G_{j}$ in $\Psi$. Then $\Psi$ is a hypertree over G. Each $G_{i}$ is a hypernode and each interface is a hyperlink.

Each hyperlink serves as the information channel between agents connected and is referred to as an agent interface. To allow efficient and exact inference, each hyperlink should render the subdomains connected conditionally independent. It can be shown (by extending results in [15]) that this implies the following structural condition. 
Definition 2 Let $G$ be a directed graph such that a hypertree over $G$ exists. $A$ node $x$ contained in more than one subgraph with its parents $\pi(x)$ in $G$ is a d-sepnode if there exists one subgraph that contains $\pi(x)$. An interface $I$ is a $\boldsymbol{d}$-sepset if every $x \in I$ is a d-sepnode.

The overall structure of an MAMSBN is a hypertree MSDAG:

Definition 3 A hypertree $\boldsymbol{M S D A G} \mathcal{D}=\bigsqcup_{i} D_{i}$, where each $D_{i}$ is a $D A G$, is a connected DAG such that (1) there exists a hypertree $\psi$ over $\mathcal{D}$, and (2) each hyperlink in $\psi$ is a d-sepset.

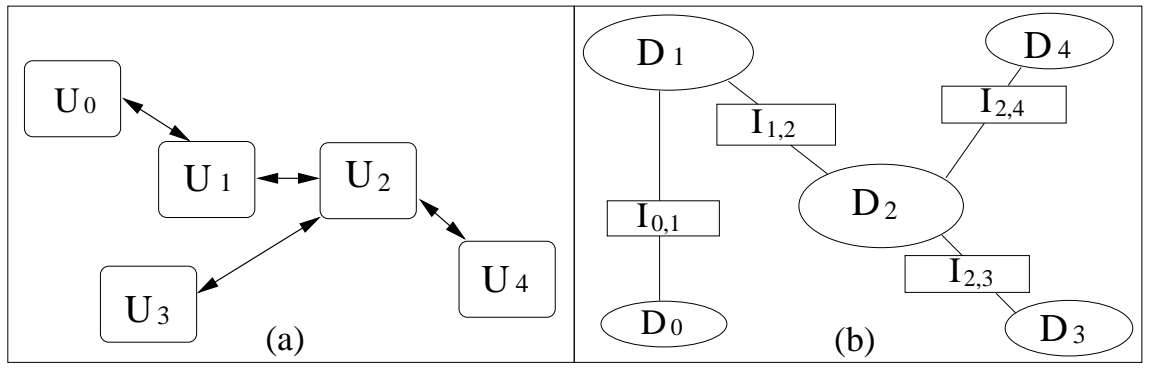

Fig. 1.1. (a) A digital system of five components. (b) The hypertree modeling.

Table 1.1. Agent communication interfaces.

\begin{tabular}{cl}
\hline Interface & Interface Composition \\
\hline$I_{0,1}$ & $\left\{a_{0}, b_{0}, c_{0}, e_{0}, f_{0}, g_{1}, g_{2}, x_{3}, z_{2}\right\}$ \\
$I_{1,2}$ & $\left\{g_{7}, g_{8}, g_{9}, i_{0}, k_{0}, n_{0}, o_{0}, p_{0}, q_{0}, r_{0}, t_{2}, y_{2}, z_{4}\right\}$ \\
$I_{2,3}$ & $\left\{a_{2}, b_{2}, d_{1}, d_{2}, d_{3}, s_{0}, u_{0}, w_{0}, x_{0}, y_{0}, z_{0}\right\}$ \\
$I_{2,4}$ & $\left\{e_{2}, h_{2}, i_{2}, j_{2}, t_{4}, t_{5}, t_{7}, w_{2}, x_{4}, y_{4}, z_{5}\right\}$ \\
\hline
\end{tabular}

As a small example, Figure 1.1 (a) shows a digital system with five components $U_{i}(i=0, \ldots, 4)$. Although how components are interfaced, as shown in (a), and the set of interface variables, as shown in Table 1.1, are known to the system integrator, internal details of each component are proprietary. To give readers a concrete idea on the scenario, a centralized perspective of the digital system is shown in Figure 1.2.

The subnets for agents $A_{1}$ and $A_{2}$ are shown in Figures 1.3 and 1.4, where each node is labeled by the variable name and an index. The agent interface $I_{1,2}$ between them contains 13 variables and is a d-sepset. For instance, the 


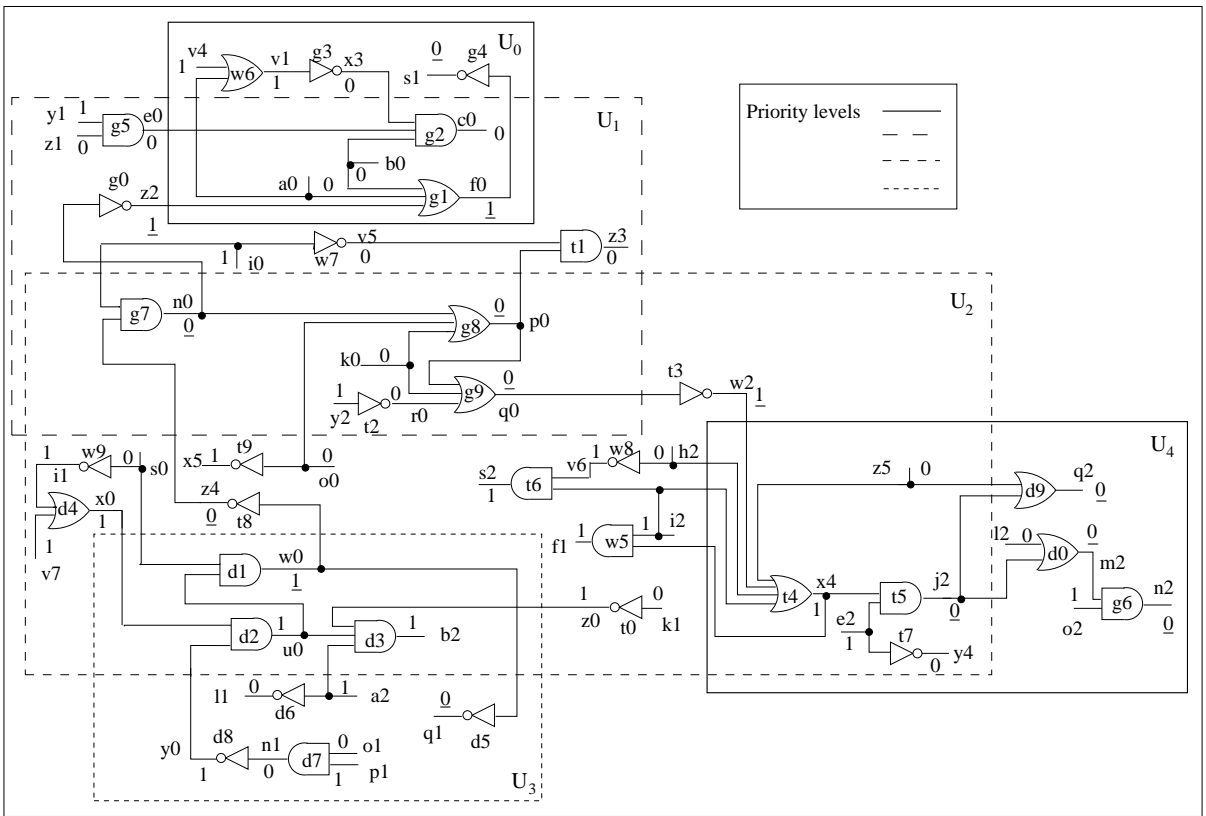

Fig. 1.2. A digital system.

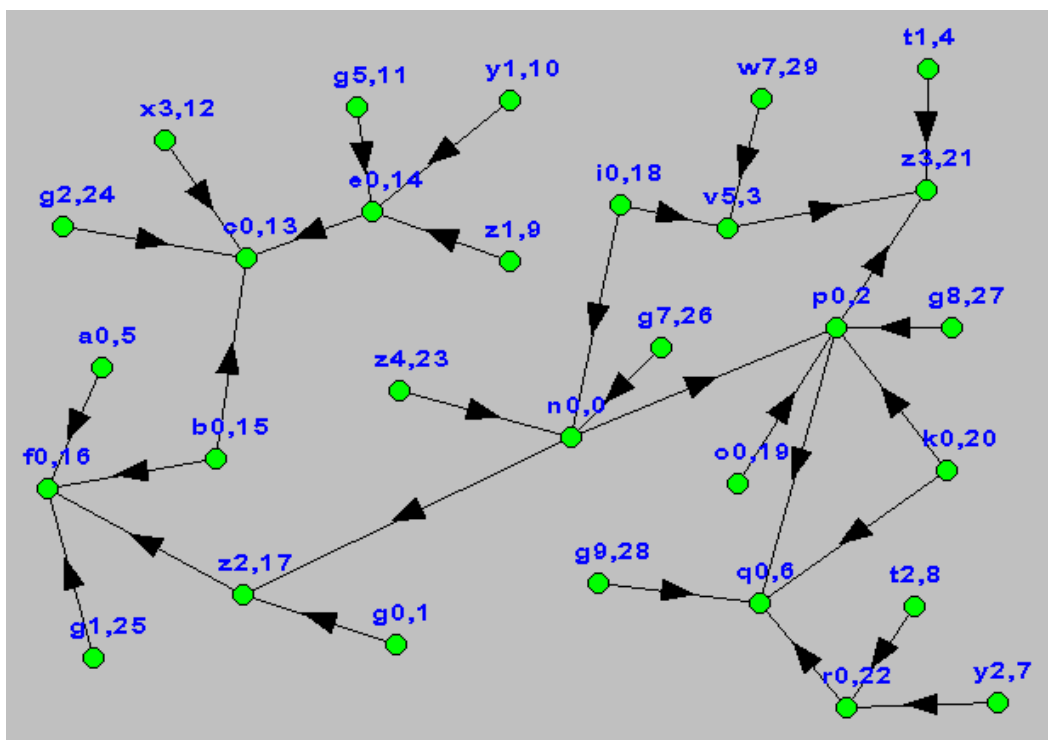

Fig. 1.3. The subnet $D_{1}$ for $U_{1}$. 
parents of $z_{4}$ are all contained in $D_{2}$, while those of $n_{0}$ are contained in both $D_{1}$ and $D_{2}$.

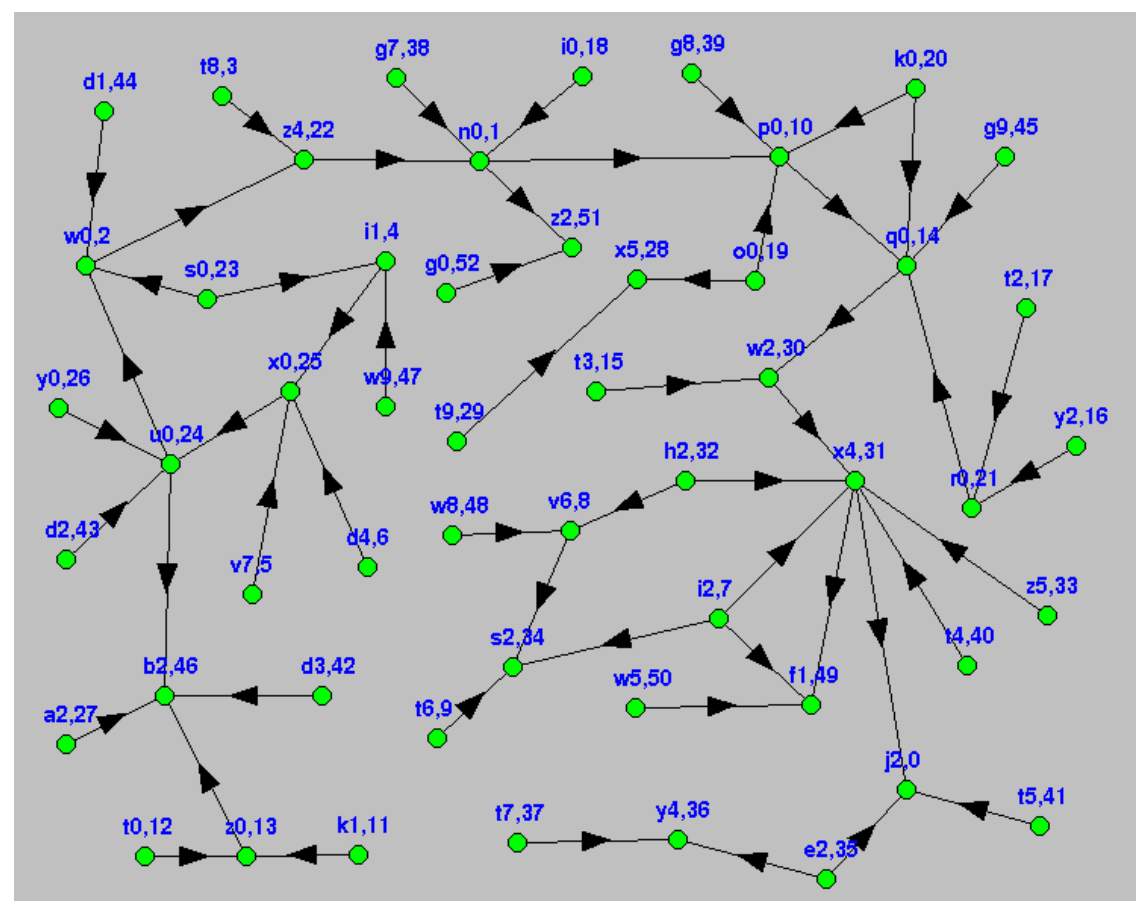

Fig. 1.4. The subnet $D_{2}$ for $U_{2}$.

In an MAMSBN integrated from agents from different vendors, no agent has the perspective of Figure 1.2, nor the simultaneous knowledge of $D_{1}$ and $D_{2}$. Only the nodes in an agent interface are public. All other nodes in a subnet are private and known to the corresponding agent only. This forms the constraint of many operations in an MAMSBN, e.g., triangulation [17] and communication [16]. Using these operations, agents can reason about their environment probabilistically based on local observations and limited communication. More formal details on MAMSBNs can be found in references noted above.

\subsection{The issue of cooperative verification}

Each agent interface in an MAMSBN should be a d-sepset (Def. 2). When an MAS is integrated from independently developed agents, there is no guarantee 
that this is the case. Blindly performing MAMSBN operations on the MAS would result in incorrect inference. Hence, agent interfaces need to be verified.

An agent interface is a d-sepset if every public node in the interface is a d-sepnode. However, whether a public node $x$ in an interface $I$ is a d-sepnode cannot be determined by the pair of local graphs interfaced with $I$. It depends on whether there exists a local DAG that contains all parents $\pi(x)$ of $x$ in $G$. Any local DAG that shares $x$ may potentially contain some parent nodes of $x$. Some parent nodes of $x$ are public, but others are private. For agent privacy, it is desirable not to disclose parentship. Hence, we cannot send the parents of $x$ in each agent to a single agent for d-sepnode verification. Cooperation among all agents whose subdomains contain $x$ or parents of $x$ is required to verify whether $x$ is a d-sepnode. We refer to the unverified structure of an MAS as a hypertree DAG union.

In presenting our method, we will illustrate using examples. Although MAMSBNs are intended for large problem domains, many issues in this paper can be demonstrated using examples of much smaller scale. Hence, we will do so for both comprehensibility as well as space. Readers should keep in mind that these examples do not reflect the scales to which MAMSBNs are applicable. Due to space limit, proofs for some formal results are omitted.

\subsection{Checking private parents}

A public node $x$ in a hypertree DAG union $G$ may have public or private parents or both. Three cases regarding its private parents are possible: more than one local DAG (Case 1), exact one local DAG (Case 2), or no local DAG (Case 3) contains private parents of $x$. The following proposition shows that the d-sepset condition is violated in Case (1).

Proposition 4 Let a public node $x$ in a hypertree $D A G$ union $G$ be a dsepnode. Then no more than one local DAG of $G$ contains private parent nodes of $x$.

Proof: Assume that two or more local DAGs contain private parent nodes of $x$. Let $y$ be a private parent of $x$ contained in a local DAG $G_{i}$ and $z$ be a private parent of $x$ contained in $G_{j}(i \neq j)$. Then there cannot be any one local DAG that contains both $y$ and $z$. Hence no local DAG contains all parents of $x$, and $x$ is not a d-sepnode by Def. 2, which is a contradiction.

Figure 1.5 shows how this result can be used to detect non-d-sepnodes. We refer to the corresponding operation as CollectPrivateParentInfo. To verify if the public node $j$ is a d-sepnode, suppose that agents perform a rooted message passing (shown by arrows in (a)). Agent $A_{4}$ sends a count 1 to $A_{3}$, signifying that it has private parents of $j$. $A_{3}$ has no private parents of $j$. It forms its own count 0 , adds the count from $A_{4}$ to its own, and sends the 


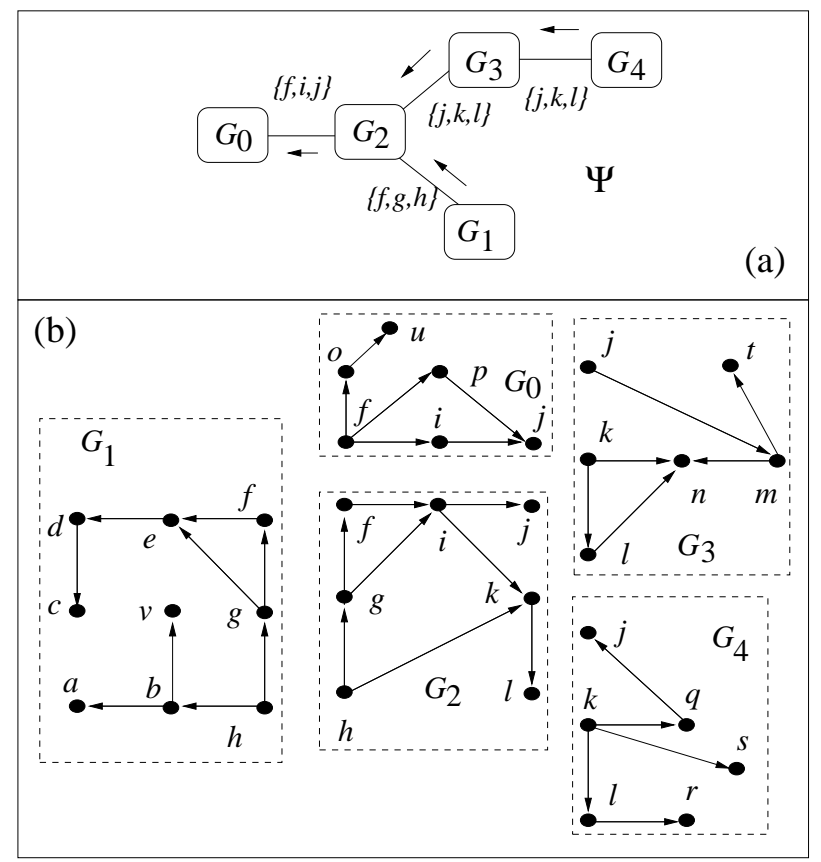

Fig. 1.5. A hypertree DAG union with the hypertree in (a) and local DAGs in (b).

result 1 to $A_{2}$. Because $A_{1}$ does not contain $j$, it does not participate in this operation. Hence, $A_{2}$ receives a message only from $A_{3}$. Because $A_{2}$ has only a public parent $i$ of $j$, it forms its own count 0 , adds the count from $A_{3}$ to its own, and sends the result 1 to $A_{0}$. Upon receiving the message, $A_{0}$ forms its own count 1 , for it has a private parent $p$ of $j$. It adds the count from $A_{2}$ to obtain 2 and the message passing halts. The final count signifies that there are two agents which contain private parents of $j$. Hence, $j$ is a non-d-sepnode and the hypertree DAG union has violated the d-sepset condition.

\subsection{Processing public parents}

If CollectPrivateParentInfo on a public node $x$ results in a final count less than or equal to 1 , then no more than one agent contains private parents of $x$ (Cases (2) and (3) above). The hypertree DAG union $G$, however, may still violate the d-sepset condition. Consider the example in Figure 1.6. The public nodes are $w, x, y, z$. No local DAG has any private parent of $x$ or $z$. Only $G_{0}$ has a private parent of $y$, and only $G_{2}$ has a private parent of $w$. Hence, CollectPrivateParentInfo will produce a final count $\leq 1$ for each of $w, x, y, z$. However, no single local DAG contains all parents of $x$ : $\pi(x)=\{w, y\}$. Therefore, $x$ is not a d-sepnode according to Def. 2 and none of the agent interfaces is a d-sepset. 


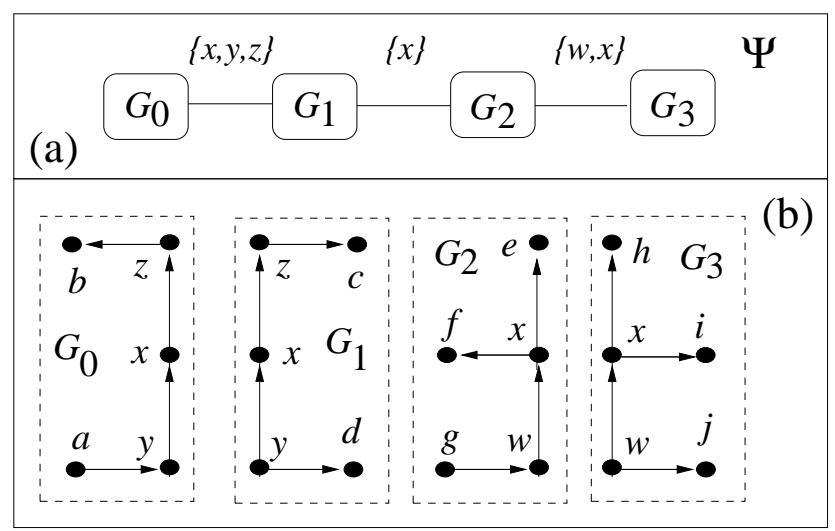

Fig. 1.6. A hypertree DAG union $G$ with the hypertree in (a) and local DAGs in (b).

The example illustrates that final counts from CollectPrivateParentInfo only provide a necessary condition for d-sepset verification. To determine if $G$ satisfies the d-sepset condition conclusively, agents still need to further process the public parents of public nodes.

First, we consider Case 3, where no local DAG contains private parents of $x$. Case 2 will be considered in Section 1.6.

\subsubsection{Public parent sequence}

We propose the following concept called public parent sequence to describe the distribution of public parents $\pi(x)$ of a public node $x$ on a hyperchain DAG union denoted as $\left\langle G_{0}, G_{1}, \ldots, G_{m}\right\rangle$. We use $X \bowtie Y$ to denote that sets $X$ and $Y$ are incomparable (neither is the subset of the other).

Definition 5 Let $\left\langle G_{0}, G_{1}, \ldots, G_{m}\right\rangle$ ( $m \geq 2$ ) be a hyperchain of local DAGs, where $x$ is a public node, each $G_{i}$ contains either $x$ or some parents of $x$, and all parents of $x$ are public. Denote the parents of $x$ that $G_{i}(0<i<m)$ shares with $G_{i-1}$ and $G_{i+1}$ by $\pi_{i}^{-}(x)$ and $\pi_{i}^{+}(x)$, respectively. Denote the parents of $x$ that $G_{m}$ shares with $G_{m-1}$ by $\pi_{m}^{-}(x)$. Then the sequence

$$
\left(\pi_{1}^{-}(x), \pi_{2}^{-}(x), \ldots, \pi_{m}^{-}(x)\right)
$$

is the public parent sequence of $x$ on the hyperchain. The sequence is classified into the following types, where $0<i<m$ :

Identical For each $i, \pi_{i}^{-}(x)=\pi_{i}^{+}(x)$.

Increasing For each $i, \pi_{i}^{-}(x) \subseteq \pi_{i}^{+}(x)$, and there exists $i$ such that $\pi_{i}^{-}(x) \subset$ $\pi_{i}^{+}(x)$.

Decreasing For each $i, \pi_{i}^{-}(x) \supseteq \pi_{i}^{+}(x)$, and there exists $i$ such that $\pi_{i}^{-}(x) \supset$ $\pi_{i}^{+}(x)$. 
Concave One of the following holds:

1. For $m \geq 3$, there exists $i$ such that the subsequence $\left(\pi_{1}^{-}(x), \ldots, \pi_{i}^{-}(x)\right)$ is increasing and the subsequence $\left(\pi_{i}^{-}(x), \ldots, \pi_{m}^{-}(x)\right)$ is decreasing.

2. There exists $i$ such that $\pi_{i}^{-}(x) \bowtie \pi_{i+1}^{-}(x)$; the preceding subsequence $\left(\pi_{1}^{-}(x), \ldots, \pi_{i}^{-}(x)\right)$ is trivial $(i=1)$, increasing, or identical; and the trailing subsequence $\left(\pi_{i+1}^{-}(x), \ldots, \pi_{m}^{-}(x)\right)$ is trivial $(i=m-1)$, decreasing, or identical.

Wave One of the following holds:

1. There exists $i$ such that $\pi_{i}^{-}(x) \supset \pi_{i}^{+}(x)$ and $j>i$ such that either $\pi_{j}^{-}(x) \subset \pi_{j}^{+}(x)$ or $\pi_{j}^{-}(x) \bowtie \pi_{j}^{+}(x)$.

2. There exists $i$ such that $\pi_{i}^{-}(x) \bowtie \pi_{i}^{+}(x)$ and $j>i$ such that either $\pi_{j}^{-}(x) \subset \pi_{j}^{+}(x)$ or $\pi_{j}^{-}(x) \bowtie \pi_{j}^{+}(x)$.

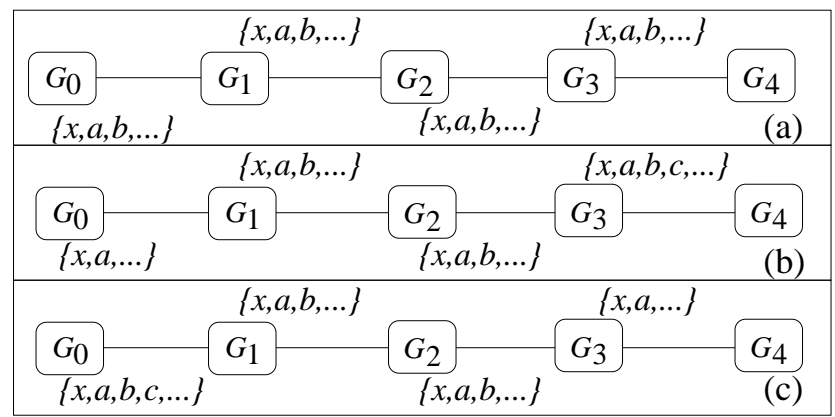

Fig. 1.7. Public parent sequences. (a) An identical sequence. (b) An increasing sequence. (c) A decreasing sequence.

Figure 1.7 illustrates the first three sequence types, where only $x$ and its parents are shown explicitly in each agent interface. Identical sequence is illustrated in (a). Each $G_{i}$ contains $\pi(x)=\{a, b\}$, and hence $x$ is a d-sepnode. Increasing sequence is exemplified in (b). From $i=1$ to $m$, each $G_{i}$ contains either the identical public parents of $x$ or more. Because $G_{m}$ contains $\pi(x)$, $x$ is a d-sepnode. Decreasing sequence is exemplified in (c). It is symmetric to the increasing sequence; $G_{0}$ contains $\pi(x)$ and $x$ is a d-sepnode.

For Concave sequence, some parents of $x$ appear in the middle of the hyperchain but not on either end. Figure 1.8 illustrates two possible cases. In (a), the parent $b$ of $x$ is contained in $G_{1}, G_{2}$, and $G_{3}$ but disappears in $G_{0}$ and $G_{4}$ and $c$ is contained in $G_{2}$ and $G_{3}$ but disappears in $G_{0}, G_{1}$, and $G_{4}$. Two local DAGs $\left(G_{2}\right.$ and $\left.G_{3}\right)$ in the middle of the hyperchain contain $\pi(x)$, and hence $x$ is a d-sepnode. In (b), an increasing subsequence ends at $\pi_{2}^{-}(x)$, and a decreasing subsequence starts at $\pi_{3}^{-}(x)$ with $\pi_{2}^{-}(x)$ and $\pi_{3}^{-}(x)$ incomparable. Because $G_{2}$ contains $\pi(x), x$ is a d-sepnode.

Figure 1.9 illustrates two possible cases of Wave sequence. In (a), a parent $d$ of $x$ appears at one end of the hyperchain, another parent $c$ appears at the 


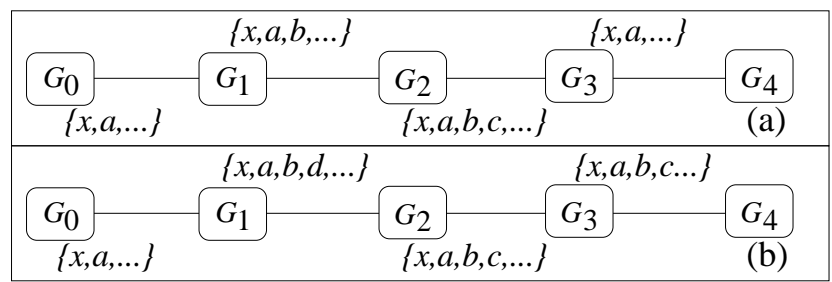

Fig. 1.8. Concave parent sequences.

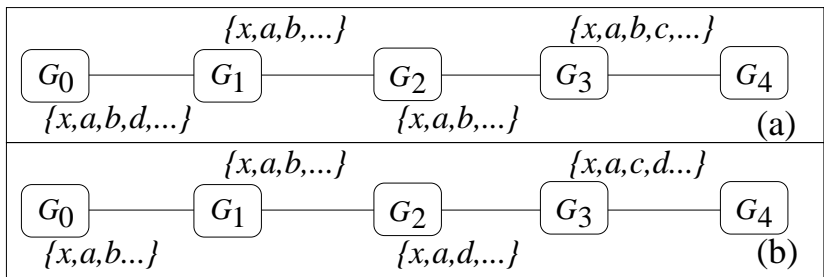

Fig. 1.9. Wave parent sequences.

other end, and they disappear in the middle of the hyperchain. In other words, we have $\pi_{1}^{-}(x) \supset \pi_{1}^{+}(x)$ and $\pi_{3}^{-}(x) \subset \pi_{3}^{+}(x)$. No local DAG contains all parents of $x$, and hence $x$ is not a d-sepnode. In (b), we have $\pi_{2}^{-}(x)$ and $\pi_{2}^{+}(x)$ being incomparable and $\pi_{3}^{-}(x) \subset \pi_{3}^{+}(x)$.

The following theorem states that the five parent sequences are exhaustive. They are also necessary and sufficient to identify d-sepnode.

Theorem 6 Let $x$ be a public node in a hyperchain $\left\langle G_{0}, G_{1}, \ldots, G_{m}\right\rangle$ of local $D A G s$ with $\pi(x)$ being the parents of $x$ in all DAGs, where no parent of $x$ is private and each local DAG contains either $x$ or some parents of $x$.

1. There exists one local DAG that contains $\pi(x)$ if and only if the public parent sequence of $x$ on the hyperchain is identical, increasing, decreasing, or concave.

2. There exists no local DAG that contains $\pi(x)$ if and only if the public parent sequence of $x$ on the hyperchain is of the wave type.

Proof:

We prove the statement 1 first:

[Sufficiency] If the sequence type is identical, then every local DAG contains $\pi(x)$. If the type is increasing, then at least $G_{m}$ contains $\pi(x)$. If the type is decreasing, then at least $G_{0}$ contains $\pi(x)$. If the type is concave, for Case (1) (see Definition 5), both $G_{i}$ and $G_{i-1}$ contain $\pi(x)$. For Case (2), $G_{i}$ contains $\pi(x)$.

[Necessity] Suppose that there exists a local DAG that contains $\pi(x)$. We show that the parent sequence of $x$ is identical, increasing, decreasing or concave. 
If every local DAG contains $\pi(x)$, then $\pi_{j}^{-}(x)=\pi_{j}^{+}(x)$ for each $j$ and the sequence is identical. Otherwise, if $G_{0}$ contains $\pi(x)$, then $\pi_{j}^{-}(x) \supseteq \pi_{j}^{+}(x)$ for each $j$ and the sequence is decreasing. Otherwise, if $G_{m}$ contains $\pi(x)$, then $\pi_{j}^{-}(x) \subseteq \pi_{j}^{+}(x)$ for each $j$ and the sequence is increasing.

Otherwise, if both $G_{i}$ and $G_{i-1}$ contain $\pi(x)$ for some $i(2 \leq i \leq m-1)$, then $\pi_{j}^{-}(x) \subseteq \pi_{j}^{+}(x)$ for each $j \leq i-1$ and the subsequence $\left(\pi_{1}^{-}(x), \ldots, \pi_{i}^{-}(x)\right)$ is increasing, and $\pi_{j}^{-}(x) \supseteq \pi_{j}^{+}(x)$ for each $j \geq i$ and the subsequence $\left(\pi_{i}^{-}(x), \ldots, \pi_{m}^{-}(x)\right)$ is decreasing. The entire parent sequence falls under concave type Case (1).

Otherwise, if only one local DAG $G_{i}$ contains $\pi(x)$, then

$$
\pi_{i}^{-}(x) \subset \pi(x) \text { and } \pi_{i}^{+}(x) \subset \pi(x) .
$$

We show $\pi_{i}^{-}(x) \bowtie \pi_{i}^{+}(x)$ by contradiction. If they are comparable, then

$$
\text { either } \pi_{j}^{-}(x) \subseteq \pi_{j}^{+}(x) \text { or } \pi_{j}^{-}(x) \supset \pi_{j}^{+}(x) .
$$

We have

$$
\pi_{j}^{-}(x) \subseteq \pi_{j}^{+}(x) \subset \pi(x) \text { or } \pi(x) \supset \pi_{j}^{-}(x) \supset \pi_{j}^{+}(x),
$$

which implies that $\pi(x)$ contains a private parent of $x$ : a contradiction. Furthermore, the subsequence $\left(\pi_{1}^{-}(x), \ldots, \pi_{i}^{-}(x)\right)$ must be trivial, increasing, or identical, and the subsequence $\left(\pi_{i+1}^{-}(x), \ldots, \pi_{m}^{-}(x)\right)$ must be trivial, decreasing, or identical. Hence, the entire parent sequence falls under concave type Case (2).

Next, we prove the statement 2 :

[Sufficiency] Suppose that the sequence is of the wave type. For wave type Case (1) in Definition 5, we have $\pi_{i}^{-}(x) \supset \pi_{i}^{+}(x)$. It implies that $G_{i-1}$ and $G_{i}$ contain a parent, say $y$, of $x$ that is not contained in $G_{i+1}$. It cannot be contained in any $G_{k}$ where $k>i+1$ owing to the hyperchain. If $\pi_{j}^{-}(x) \subset$ $\pi_{j}^{+}(x)$ holds, then $G_{j+1}$ and $G_{j}$ contain a parent, say $z$, of $x$ that is not contained in $G_{j-1}$. It cannot be contained in any $G_{k}$, where $k<j-1$. In summary, only local DAGs $G_{0}, \ldots, G_{i}$ may contain $y$ (not necessarily all of them contain $y$ ), and only $G_{j}, \ldots, G_{m}$ may contain $z$. Because $i<j$, no local DAG contains both $y$ and $z$.

If $\pi_{j}^{-}(x) \bowtie \pi_{j}^{+}(x)$, it implies that $G_{j+1}$ and $G_{j}$ contain a parent, say $z$, of $x$ that is not contained in $G_{j-1}$, and $G_{j-1}$ and $G_{j}$ contains a parent, say $w$, of $x$ that is not contained in $G_{j+1}$. Because the same condition as above holds, no local DAG contains both $y$ and $z$. For wave type Case (2), the same conclusion can be drawn.

[Necessity] Suppose that no local DAG contains $\pi(x)$. Then there exists a pair of local DAGs $G_{i}$ and $G_{j}(i<j)$ such that the following hold:

1. The DAG $G_{i}$ contains a parent, say $y$, of $x$ that is not contained in $G_{j}$, and $G_{i}$ is the closest such local DAG to $G_{j}$ on the hyperchain. 
2. The DAG $G_{j}$ contains a parent, say $z$, of $x$ that is not contained in $G_{i}$, and $G_{j}$ is the closest such local DAG to $G_{i}$ on the hyperchain.

3. No other local DAGs contain both $y$ and $z$.

Clearly, we have either $\pi_{i}^{-}(x) \supset \pi_{i}^{+}(x)$ or $\pi_{i}^{-}(x) \bowtie \pi_{i}^{+}(x)$, and either $\pi_{j}^{-}(x) \subset$ $\pi_{j}^{+}(x)$ or $\pi_{j}^{-}(x) \bowtie \pi_{j}^{+}(x)$. Hence, the sequence is of the wave type.

\subsubsection{Cooperative verification in hyperchain}

To identify the sequence type by cooperation, agents on the hyperchain pass messages from one end to the other, say, from $G_{m}$ to $G_{0}$. Each agent $A_{i}$ passes a message to $A_{i-1}$ formulated based on the message that $A_{i}$ receives from $A_{i+1}$ as well as on the result of comparison between $\pi_{i}^{-}(x)$ and $\pi_{i}^{+}(x)$. Note that $A_{i+1}$ is undefined for $A_{m}$.

We partition the five public parent sequence types into three groups and associate each group with a message coded using an integer, as shown in Table 1.2 .

Table 1.2. Message code according to public parent sequence types

\begin{tabular}{cc}
\hline type group & code \\
\hline decreasing or identical & -1 \\
increasing or concave & 1 \\
wave & 0 \\
\hline
\end{tabular}

Agents pass messages according to the algorithm CollectPublicParentInfoOnChain as defined below:

\section{Algorithm 1 (CollectPublicParentInfoOnChain)}

If $A_{i+1}$ is undefined, agent $A_{i}$ passes -1 to $A_{i-1}$. Otherwise, $A_{i}$ receives a message from $A_{i+1}$, compares $\pi_{i}^{-}(x)$ with $\pi_{i}^{+}(x)$, and sends its own message according to one of the following cases:

1. The message received is -1 :

If $\pi_{i}^{-}(x) \supseteq \pi_{i}^{+}(x), A_{i}$ passes -1 to $A_{i-1}$.

Otherwise, $A_{i}$ passes 1 to $A_{i-1}$.

2. The message received is 1 :

If $\pi_{i}^{-}(x) \subseteq \pi_{i}^{+}(x), A_{i}$ passes 1 to $A_{i-1}$.

Otherwise, $A_{i}$ passes 0 to $A_{i-1}$.

3. The message received is $0: A_{i}$ passes 0 to $A_{i-1}$. 
We demonstrate how agents cooperate using examples in Figures 1.7 through 1.9. In Figure 1.7 (a), -1 is sent from $A_{4}$ to $A_{3}$ and is passed along by each agent until $A_{0}$ receives it. Interpreting the message code, $A_{0}$ concludes that the parent sequence is either identical or decreasing. Because the actual sequence is identical, the conclusion is correct.

In (b), $A_{3}$ receives -1 from $A_{4}$ and sends 1 to $A_{2}$. Afterwards, 1 is passed all the way to $A_{0}$, which determines that the sequence is either increasing (actual type) or concave.

In (c), -1 is sent by each agent. The conclusion drawn by $A_{0}$ is to classify the type of sequence as either identical or decreasing (actual type).

In Figure 1.8 (a), $A_{3}$ receives -1 from $A_{4}$ and sends -1 to $A_{2}$. Agent $A_{2}$ sends 1 to $A_{1}$, which passes it to $A_{0}$. Agent $A_{0}$ then concludes that the sequence type is either increasing or concave, where concave is the actual type. In (b), -1 is sent from $A_{4}$ to $A_{3}$ and then to $A_{2}$. Agent $A_{2}$ sends 1 to $A_{1}$, which is passed to $A_{0}$.

In Figure 1.9 (a), $A_{3}$ receives -1 from $A_{4}$ and sends 1 to $A_{2}$. Agent $A_{2}$ passes 1 to $A_{1}$, which in turn sends 0 to $A_{0}$. Agent $A_{0}$ then interprets the sequence type as a wave, which matches the actual type. In (b), $A_{3}$ receives -1 from $A_{4}$ and sends 1 to $A_{2}$. Agent $A_{2}$ sends 0 to $A_{1}$, which passes 0 to $A_{0}$.

In summary, each agent on the hyperchain can pass a code message formulated based on the message it receives and the comparison of the public parents it shares with the adjacent agents. The message passing starts from one end of the hyperchain and the type of the public parent sequence can be determined by the agent in the other end. In this cooperation, no agent needs to disclose its internal structure.

\subsubsection{Cooperative verification in hypertree}

We investigate the issue in a general hypertree, and let agents to cooperate in a similar way as in a hyperchain. However, the message passing is directed towards an agent acting as the root of the hypertree.

Consider first the case in which the root agent $A_{i}$ has exactly two adjacent agents $A_{1}$ and $A_{2}$. If an agent $A_{i}$ has a downstream adjacent agent $A_{k}$, we denote the parents of $x$ that $A_{i}$ shares with $A_{k}$ by $\pi_{k}(x)$. In Section 1.5.2, $A_{i}$ receives message from $A_{1}$ and sends message to $A_{2}$, so the only information that agent $A_{i}$ needs to process is the message received from $A_{1}$. Here, $A_{i}$ receives messages from both $A_{1}$ and $A_{2}$. Thus, $A_{i}$ has three pieces of information: two messages received from adjacent agents and a comparison between $\pi_{1}(x)$ and $\pi_{2}(x)$. The key to determine whether $x$ is a d-sepnode is to detect whether its public parent sequence along any hyperchain, on the hypertree, is the wave type. A wave sequence can be detected based on one message received by $A_{i}$ only (when the hyperchain from $A_{i}$ to a terminal agent is a wave), or if not sufficient based on both messages received, or if still not sufficient based in addition on the comparison between $\pi_{1}(x)$ and $\pi_{2}(x)$. 
The idea can be applied to a general hypertree where $A_{i}$ has any finite number of adjacent agents. Now $A_{i}$ must take into account the three pieces of information for each pair of adjacent agents. Consider the hypertree in

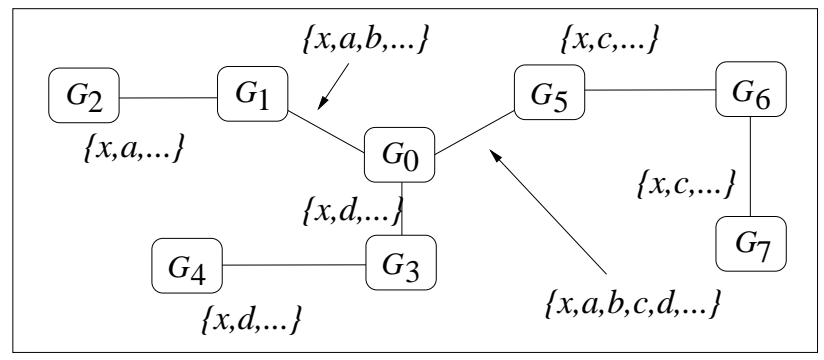

Fig. 1.10. Parents $\pi(x)$ of a d-sepnode $x$ shared by local DAGs in a hypertree.

Figure 1.10. If $A_{0}$ is the root, then messages will be passed towards $A_{0}$ from terminal agents $A_{2}, A_{4}$, and $A_{7}$. After agents send messages according to CollectPublicParentInfoOnChain, $A_{0}$ receives -1 from each of $A_{1}, A_{3}$, and $A_{5}$. This implies that the parent sequence type of each hyperchain from $A_{0}$ to a terminal agent is either identical or decreasing. Hence agent $A_{0}$ can conclude that itself contains $\pi(x)$ and $x$ is a d-sepnode.

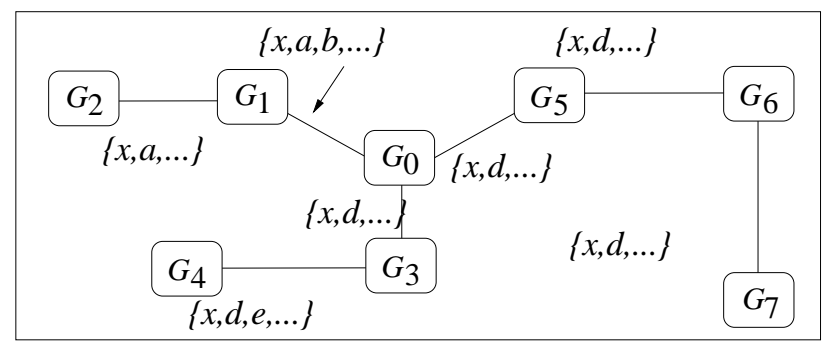

Fig. 1.11. Parents $\pi(x)$ of a non-d-sepnode $x$ shared by local DAGs in a hyperstar.

In Figure 1.11, suppose that $A_{5}$ is the root. Messages will be passed towards $A_{5}$ from terminal agents $A_{2}, A_{4}$, and $A_{7}$. Agent $A_{0}$ will receives -1 from $A_{1}$ and 1 from $A_{3}$. It realizes that each hyperchain from $A_{0}$ downstream through $A_{1}$ is either identical or decreasing and the hyperchain from $A_{0}$ downstream through $A_{3}$ is either increasing or concave. Because the messages are not sufficient to conclude, $A_{0}$ compares $\pi_{1}(x)$ with $\pi_{3}(x)$. It discovers that they are incomparable. This implies that there exist a hyperchain $H_{1}$ from $A_{0}$ downstream through $A_{1}$ and a hyperchain $H_{3}$ from $A_{0}$ downstream through $A_{3}$ such that when $H_{1}$ is joined with $H_{3}$ the resultant hyperchain has a wave parent sequence. Hence, $A_{0}$ will pass the code mes- 
sage 0 to $A_{5}$. Based on this message, the root agent $A_{5}$ concludes that $x$ is not a d-sepnode. The conclusion is correct because no local DAG contains both $a$ and $e$.

The following algorithm describes the actions a typical agent $A_{0}$ performs.

\section{Algorithm 2 (CollectPublicParentInfo(x))}

1. Receive a message $m_{i}$ from each downstream adjacent agent $A_{i}$.

2. (a) If any message is $0, A_{0}$ sends 0 to the upstream agent $A_{c}$.

(b) Otherwise, if any two messages are $1, A_{0}$ sends 0 to $A_{c}$.

(c) Otherwise, if a message $m_{i}$ is 1 , then $A_{0}$ compares $\pi_{i}(x)$ with $\pi_{j}(x)$ for each downstream adjacent agent $A_{j}$. If $j$ is found such that $\pi_{i}(x) \nsupseteq$ $\pi_{j}(x), A_{0}$ sends 0 . If not found, $A_{0}$ sends 1 .

(d) Otherwise, continue.

3. $A_{0}$ compares each $\pi_{i}(x)$ with the parents $\pi_{c}(x)$ shared with $A_{c}$. If there exists $i$ such that $\pi_{c}(x) \nsupseteq \pi_{i}(x)$, then $A_{0}$ sends 1 to $A_{c}$. Otherwise, $A_{0}$ sends -1 .

The following theorem establishes that d-sepnode condition can be verified correctly by agent cooperation through CollectPublicParentInfo.

Theorem 7 Let a hypertree of local DAGs $\left\{G_{i}\right\}$ be populated by a set of agents. Let $x$ be a public node with only public parents in the hypertree. Let agents pass messages according to CollectPublicParentInfo(x).

Then $x$ is a non-d-sepnode if and only if the root agent returns 0.

\subsection{Cooperative verification in a general hypertree}

We consider cooperative verification of the d-sepnode condition when both public and private parents of a public nodes are present. Agents who populate such a hypertree can first perform CollectprivateParentInfo to find out whether more than one local DAG contains private parents of $x$. If two or more agents are found to contain private parents of $x$, then agents can conclude, by Proposition 4, $x$ is a non-d-sepnode. If no agent is found to contain private parents of $x$, then agents can perform CollectPublicParentInfo with any agent being the root to determine if $x$ is a d-sepnode.

On the other hand, if one agent $A_{0}$ is found to contain private parents of $x$, then agents can perform CollectPublicParentInfo with $A_{0}$ being the root to determine if $x$ is a d-sepnode. Note that it is necessary for $A_{0}$ to be the root. For instance, in Figure 1.10, if $A_{2}$ is the only agent that contains the private parents of $x$, when CollectPublicParentInfo is performed with the root $A_{0}$, agent $A_{0}$ cannot conclude as in Section 1.5.3. Clearly, although $A_{0}$ contains all public parents of $x$, it does not contain the private parents of $x$. Hence, it is unknown to $A_{0}$ whether there is an agent containing all parents of $x$. In this case, it depends on whether $A_{2}$ is such an agent.

The following algorithm summarizes the method. 


\section{Algorithm 3 (VerifyDsepset)}

Let a hypertree DAG union $G$ be populated by multiple agents with one at each hypernode. For each public node $x$, agents cooperate as follows:

1. Agents perform CollectprivateParentInfo. If more than one agent is found to contain private parents of $x$, conclude that $G$ violates the $d$-sepset condition.

2. If no agent is found to contain private parents of $x$, agents perform CollectPublicParentInfo with any agent $A_{0}$ as the root. If $A_{0}$ generates the message 0, conclude that $G$ violates the $d$-sepset condition. Otherwise, conclude that $G$ satisfies the d-sepset condition.

3. If a single agent $A_{0}$ is found to contain private parents of $x$, then agents perform CollectPublicParentInfo with $A_{0}$ as the root. If $A_{0}$ generates the message -1, conclude that $G$ satisfies the $d$-sepset condition. Otherwise, conclude that $G$ violates the d-sepset condition.

It can be proven that VerifyDsepset accomplishes the intended task correctly:

Theorem 8 Let a hypertree DAG union $G$ be populated by multiple agents. After VerifyDsepset is executed in $G$, it concludes correctly with respect to whether $G$ satisfies the d-sepset condition.

\subsection{Complexity}

We show that multiagent cooperative verification by VerifyDsepset is efficient. We denote the maximum cardinality of a node adjacency in a local DAG by $t$; the maximum number of nodes in an agent interface by $k$; the maximum number of agents adjacent to any given agent on the hypertree by $s$; and the total number of agents by $n$.

Each agent may call CollectPrivateParentInfo $O(k s)$ times - one for each shared node. Each call may propagate to $O(n)$ agents. Examination of whether a shared node has private parents in a local DAG takes $O(t)$ time. Hence, the total time complexity for checking private parents is $O\left(n^{2} k s t\right)$.

Next, we consider processing of public parents after checking private parents succeeds positively. The computation time is dominated by CollectPublicParentInfo. Each agent may call CollectPublicParentInfo $O(k s)$ times. Each call may propagate to $O(n)$ agents. When processing public parent sequence information, an agent may compare $O(s)$ pairs of agent interfaces. Each comparison examines $O\left(k^{2}\right)$ pairs of shared nodes. Hence, the total time complexity for processing public parents is $O\left(n^{2} k^{3} s^{2}\right)$. The overall complexity of VerifyDsepset is $O\left(n^{2}\left(k^{3} s^{2}+k s t\right)\right)$ and the computation is efficient. 


\subsection{Alternative Methods of Verification}

Some alternative verification methods to VerifyDsepset are worth considering. We analyze alternative methods that deviate from VerifyDsepset around two aspects: first, verification by centralizing the parent set information; and second, verification by asynchronous message passing.

According to Definition 2, to determine whether a public node $x$ is a dsepnode, one needs to know whether a local DAG contains $\pi(x)$. For Case 3 of Section 1.4, $\pi(x)$ contains only public parents. Hence, it appears that a direct test whether there exists a local DAG containing $\pi(x)$ can be employed.

To put this idea to work, for each such public node $x$, one needs to centralize the information on $\pi(x)$ somehow. This can be done in at least two ways. The first is to let agents propagate the information on public parents of $x$ through the hypertree. Two passes (inwards and then outwards) are sufficient so that every agent knows about $\pi(x)$. Each can then determine whether $\pi(x)$ is contained locally.

The drawback of this alternative is that information on each element in $\pi(x)$ is disclosed to agents that may not share the element. Note that $\pi(x)$ is public only in the sense that each variable in $\pi(x)$ is shared by two or more agents. An agent that shares one variable in $\pi(x)$ may not share another. Hence, this alternative publicizes $\pi(x)$ beyond what is necessary.

An alternative is to collect the information on $\pi(x)$ by a single agent $A$. Each agent containing $x$ needs to send information on its public parents of $x$ to $A$. After $A$ collected information on $\pi(x), A$ checks with each agent whether it contains the entire $\pi(x)$.

This method restricts the access to information on $\pi(x)$ to a single agent and is superior than the previous method as far as the privacy issue is concerned. On the other hand, VerifyDsepset does not require such a centralized agent at all. One may argue that in the construction of an MAMSBN, an integrator (Section 1.2) already knows all the public variables and is a suitable candidate for $A$. Note, however, that the integrator only needs to know what are interface variables. It does not need to know the structural (parent) information on public variables. In summary, although the alternative methods appear to be much simpler, VerifyDsepset provides the highest level of privacy for internal structural information of agents.

Next, we consider an alternative method for message passing. VerifyDsepset uses a number of rooted message propagations. For instance, CollectPrivateParentInfo shown in Figure 1.5 can be performed by first propagating a control message from the root agent $A_{0}$ (located at $G_{0}$ ) to the leaf agents $A_{1}$ and $A_{4}$, and then propagating the private parent information from $A_{1}$ and $A_{4}$ back to $A_{0}$. Alternatively, message passing in a tree structure can be performed in an asynchronous fashion such as that used in Shafer-Shenoy belief propagation [13]:

In an asynchronous message passing, each agent on the tree sends one message to each neighbor. It can send a message to a neighbor only after 
it has received a message from each other neighbor (and, in general, the message sent is dependent on the received messages). Figure 1.12 illustrates an asynchronous message passing. In (a), agents $A_{2}, A_{4}$ and $A_{5}$ are the only

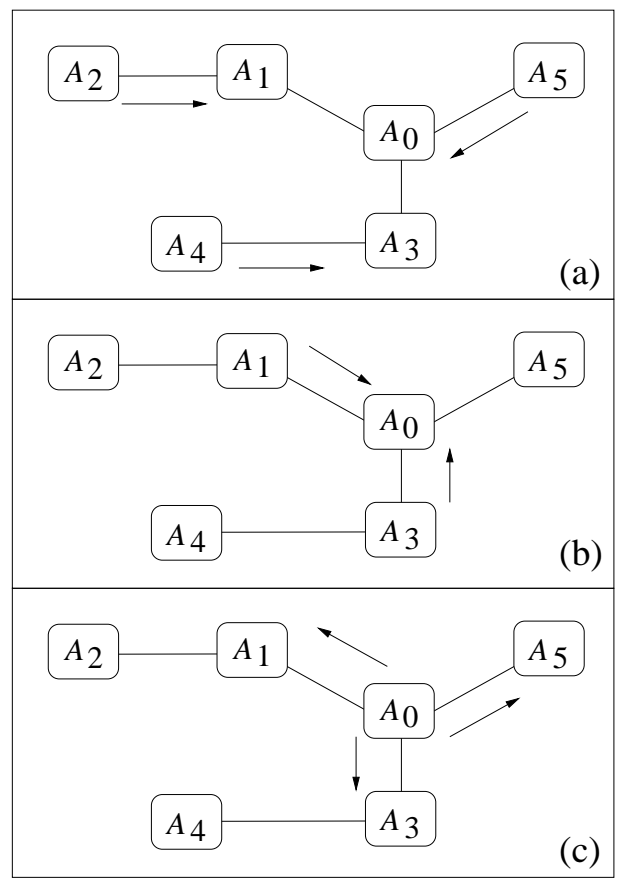

Fig. 1.12. Asynchronous message passing

ones that are able to send the message (shown by arrows). In (b), $A_{3}$ has received message from $A_{4}$ and is now ready to send to $A_{0}$. Similarly, $A_{1}$ is ready to send to $A_{0}$. In (c), $A_{0}$ has received messages from $A_{1}$ and $A_{5}$ and is ready to send to $A_{3}$. For similar reasons, it is also ready to send to $A_{1}$ and $A_{5}$. Afterwards, $A_{1}$ can send to $A_{2}$, and $A_{3}$ can send to $A_{4}$ (not shown). The asynchronous message passing is then completed.

An asynchronous message passing sends exactly the same set of messages by each agent as in a rooted message passing. Therefore, in principle, VerifyDsepset could be performed by asynchronous message passing. However, depending on whether the MAMSBN is open or closed, the rooted message passing can be more advantageous, as analyzed below.

When an MAMSBN is closed (agent membership does not change), interface verification needs to be performed once for all. For rooted message passing, a root agent needs to be selected and agreed by all (otherwise, no agent will act as the root or multiple of them will). For asynchronous message passing, all leaf agents (with exactly one neighbor) must agree on roughly 
when to start (otherwise, some may start and wait for ever for others). In either case, a value needs to be agreed upon (who is the root or when to start). For how agents can reach such an agreement, see Coulouris et al. [1]. The point is, an equivalent amount of effort is needed in either case. Therefore, there does not seem to be any reason to favor one method of message passing over the other when an MAMSBN is closed.

On the other hand, when an MAMSBN is open, verification may need to be performed again when additional agents join. For asynchronous message passing, all leaf agents must reach another agreement. For rooted message passing, as long as the previous root agent is still a member, it can continue to function as the root. No new agreement is needed. Hence, rooted message passing has an advantage over asynchronous message passing when an MAMSBN is open.

\subsection{Conclusion}

We present a method to verify agent interface in an MAS whose knowledge representation is based on MSBNs. To ensure exact, distributed probabilistic inference, agent interfaces must be d-sepsets. Using our verification method, agents only pass concise messages among them without centralized control. A message reveals only partial information about the parenthood of a public node without disclosing additional details on the agent's local DAG. Hence, the method respects agent's privacy, protects agent vendors' know-how, and promotes integration of MAS from independently developed agents.

MSBNs support both modular, exact probabilistic inference in single agent systems and exact, distributed probabilistic inference in MAS. The connection between MSBNs and OOBNs was explored by Koller and Pfeffer [6]. Although OOBNs are intended for single agent systems, the object interfaces also have to satisfy the d-sepset condition. The approach taken was to require all arcs from one network segment to another to follow the same direction. Owing to this requirement, the d-sepset condition is automatically satisfied in a hypertree DAG union. No verification is required. On the other hand, the requirement does restrict the dependency structures to a proper subset of general MSBNs. For instance, in the MAMSBN for monitoring the digital system (Figure 1.2), arcs may go either way between a pair of adjacent

local DAGs. The method presented in this paper allows agent interfaces to be verified efficiently in a general MAMSBN.

\section{Acknowledgements}

The funding support from Natural Sciences and Engineering Research Council (NSERC) of Canada to the first author is acknowledged. 


\section{References}

1. G. Coulouris, J. Dollimore, and T. Kindberg. 2001. Distributed Systems: Concepts and Design, 3rd Ed. Addison-Wesley.

2. A.F. Dragoni, P. Giorgini, and L. Serafini. 2001. Updating mental states from communication. In Intelligent Agents VII: Agent Theories, Architectures and Languages. Springer-Verlag.

3. P.J. Gmytrasiewicz and C.L. Lisetti. 2000. Using decision theory to formalize emotions for multi-agent systems. In Second ICMAS-2000 Workshop on Game Theoretic and Decision Theoretic Agents, Boston.

4. M.N. Huhns and D.M. Bridgeland. 1991. Multiagent truth maintenance. IEEE Trans. Sys., Man, and Cybernetics, 21(6):1437-1445.

5. G. Kaminka and M. Tambe. 1999. I'm ok, you're ok, we're ok: experiments in centralized and distributed socially attentive monitoring. In Proc. Inter. Conference on Automonomous Agents.

6. D. Koller and A. Pfeffer. 1997. Object-oriented Bayesian networks. In D. Geiger and P.P. Shenoy, editors, Proc. 13th Conf. on Uncertainty in Artificial Intelligence, pages 302-313, Providence, Rhode Island.

7. V.R. Lesser and L.D. Erman. 1980. Distributed interpretation: a model and experiment. IEEE Trans. on Computers, C-29(12):1144-1163.

8. C.L. Mason and R.R. Johnson. 1989. DATMS: a framework for distributed assumption based reasoning. In L. Gasser and M.N. Huhns, editors, Distributed Artificial Intelligence II, pages 293-317. Pitman.

9. P. McBurney and S. Parsons. 2001. Chance discovery using dialectical argumentation. In T. Terano, T. Nishida, A. Namatame, S. Tsumoto, Y. Ohsawa, and T. Washio, editors, New Frontiers in Artificial Intelligence, Lecture Notes in Artificial Intelligence Vol. 2253, pages 414-424. Springer-Verlag.

10. H.P. Nii. 1986. Blackboard systems: the blackboard model of problem solving and the evolution of blackboard architectures. AI Magazine, 7(2):38-53.

11. J. Pearl. 1988. Probabilistic Reasoning in Intelligent Systems: Networks of Plausible Inference. Morgan Kaufmann.

12. A. Rao and M. Georgeff. 1991. Deliberation and its role in the formation of intentions. In B. D'Ambrosio, P. Smets, and P.P. Bonissone, editors, Proc. 7th Conf. on Uncertainty in Artificial Intelligence, pages 300-307. Morgan Kaufmann.

13. G. Shafer. 1996. Probabilistic Expert Systems. Society for Industrial and Applied Mathematics, Philadelphia.

14. K.P. Sycara. 1998. Multiagent systems. AI Magazine, 19(2):79-92.

15. Y. Xiang and V. Lesser. 2000. Justifying multiply sectioned Bayesian networks. In Proc. 6th Inter. Conf. on Multi-agent Systems, pages 349-356, Boston.

16. Y. Xiang. 2000. Belief updating in multiply sectioned Bayesian networks without repeated local propagations. Inter. J. Approximate Reasoning, 23:1-21.

17. Y. Xiang. 2001. Cooperative triangulation in MSBNs without revealing subnet structures. Networks, 37(1):53-65. 